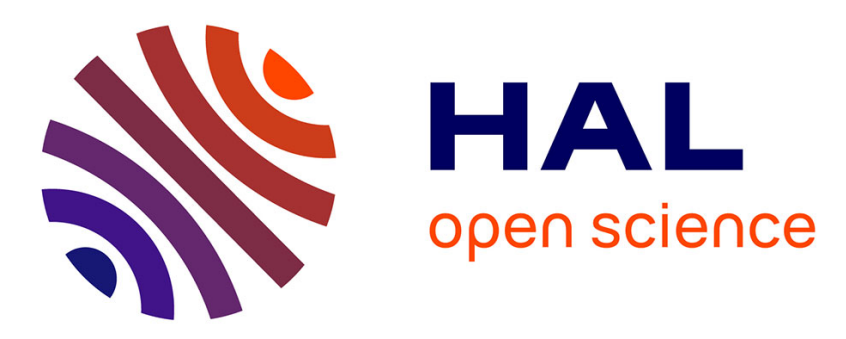

\title{
Buckling Instability Causes Inertial Thrust for Spherical Swimmers at All Scales
}

\author{
Adel Djellouli, Philippe Marmottant, Henda Djeridi, Catherine Quilliet, \\ Gwennou Coupier
}

\section{- To cite this version:}

Adel Djellouli, Philippe Marmottant, Henda Djeridi, Catherine Quilliet, Gwennou Coupier. Buckling Instability Causes Inertial Thrust for Spherical Swimmers at All Scales. Physical Review Letters, 2017, 119 (22), pp.224501. 10.1103/PhysRevLett.119.224501 . hal-01653208

\section{HAL Id: hal-01653208 \\ https://hal.science/hal-01653208}

Submitted on 1 Dec 2017

HAL is a multi-disciplinary open access archive for the deposit and dissemination of scientific research documents, whether they are published or not. The documents may come from teaching and research institutions in France or abroad, or from public or private research centers.
L'archive ouverte pluridisciplinaire HAL, est destinée au dépôt et à la diffusion de documents scientifiques de niveau recherche, publiés ou non, émanant des établissements d'enseignement et de recherche français ou étrangers, des laboratoires publics ou privés. 


\title{
Buckling Instability Causes Inertial Thrust for Spherical Swimmers at All Scales
}

\author{
Adel Djellouli, ${ }^{1}$ Philippe Marmottant, ${ }^{1}$ Henda Djeridi, ${ }^{2}$ Catherine Quilliet, ${ }^{1}$ and Gwennou Coupier ${ }^{1, *}$ \\ ${ }^{1}$ Université Grenoble Alpes, CNRS, LIPhy, F-38000 Grenoble, France \\ ${ }^{2}$ Université Grenoble Alpes, Grenoble INP, CNRS, LEGI, F-38000 Grenoble, France
}

(Received 19 June 2017; published 27 November 2017)

\begin{abstract}
Microswimmers, and among them aspirant microrobots, generally have to cope with flows where viscous forces are dominant, characterized by a low Reynolds number (Re). This implies constraints on the possible sequences of body motion, which have to be nonreciprocal. Furthermore, the presence of a strong drag limits the range of resulting velocities. Here, we propose a swimming mechanism which uses the buckling instability triggered by pressure waves to propel a spherical, hollow shell. With a macroscopic experimental model, we show that a net displacement is produced at all Re regimes. An optimal displacement caused by nontrivial history effects is reached at intermediate Re. We show that, due to the fast activation induced by the instability, this regime is reachable by microscopic shells. The rapid dynamics would also allow high-frequency excitation with standard traveling ultrasonic waves. Scale considerations predict a swimming velocity of order $1 \mathrm{~cm} / \mathrm{s}$ for a remote-controlled microrobot, a suitable value for biological applications such as drug delivery.
\end{abstract}

DOI: 10.1103/PhysRevLett.119.224501

Besides their playful aspect, artificial microswimmers present undeniable fundamental and practical interests, mostly driven by a constant race toward increasing miniaturization with potential applications such as targeted drug delivery. Comprehensive studies aim to identify the efficient strategies for small-scale displacement in liquids [1-7], which can possibly be exploited for the conception of synthetic microswimmers. Sticking to the strict definition of swimming as performing a displacement induced by body deformation, quite a few realizations of synthetic microswimmers can be found in literature [8-12]. A growing attention toward the simplicity of their fabrication [13-15] opens possibilities for transfer in the industrial arena. The two main external sources of power are magnetic $[10,11,13]$ and acoustic [12,14,15] fields, which are probably more suitable for medical applications and less expensive. The major conceptual difficulty lies in the low-Reynolds-number flows usually associated with microscopic scales; the scallop theorem [16] then imposes that a nonzero displacement may only occur via a nonreciprocal succession of shapes. Except in chiral systems $[10,11]$, this necessary condition requires at least 2 degrees of freedom, which commonly implies two control parameters. Such heavy double steering could indeed be bypassed if flow rates can be rendered high enough so that inertia could no longer be neglected, or if any hysteresis in the deformation "naturally" prevents reciprocity.

We suggest fulfilling these two conditions together with simple spherical colloidal shells full of air, which are microscopic objects quite easy to manufacture [17,18]. Deflation from a spherical geometry occurs via buckling, which is a subcritical instability likely to provide both swiftness and hysteresis during a deflation-reinflation cycle driven by a single scalar control parameter: pressure. We investigate the swimming that results from these deformations thanks to macroscopic shells placed in a set of fluids with varying viscosity so that relevant dimensionless numbers could be kept unchanged from the microscopic scale.

Design and actuation of the swimmer.-The swimmer was a hollow sphere of thickness $d$ and external radius $R+d / 2=25 \mathrm{~mm}$, made in an elastomer of Young modulus $E=0.5 \mathrm{MPa}$. The pressure inside the shell

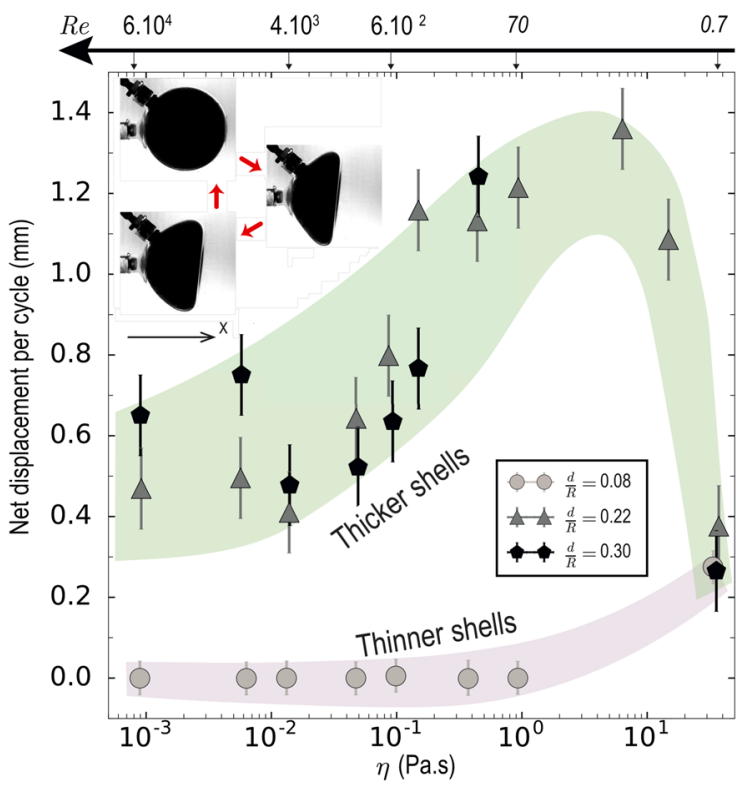

FIG. 1. Inset: Illustration of the deformation cycle. Main figure: Displacement in the $x$ direction after one deformation cycle for three different $d / R$ ratios, as a function of fluid viscosity. The indicated Reynolds numbers correspond to $d / R=0.22$. Green (top) shaded area highlights the high-elastic-energy regime while the low-elasticenergy regime is indicated by the pink (bottom) shaded area. 
was controlled by a pressure controller while the shell was attached to a frictionless rail. A weak spot for buckling was oriented in the rail direction ( $x$ axis, inset of Fig. 1). Deformation without displacement was also studied by controlling the external pressure to be closer to the anticipated microscopic situation (activation by pressure waves). In that case, the shell was immersed in a pressurized tank (see [19] for more details on the method).

After a pressure cycle of sufficient amplitude so that buckling occurs, the shell and its support always move in the same direction, regardless of the shell thickness and the fluid viscosity (Fig. 1). Deeper insight into this swimming motion requires us to first to focus on the deformation dynamics.

Shell deformation cycle.-A stress-free elastic spherical shell of radius $R$ and thickness $d$ submitted to an outsideinside pressure difference $\Delta P$ first shrinks while keeping its spherical symmetry, which corresponds to a quasilinear relationship between $\Delta P$ and the volume variation [path A-B in Fig. 2(a)] [20,21]. Then, over a threshold pressure difference $\Delta P_{C} \simeq E(d / R)^{2} \quad[20,22-24]$, an instability occurs toward a highly deflated conformation with a depression of extent $\sim R[20,24]$. In practice, the final state depends on the possibility to compress the inner medium [see, e.g., $\mathrm{C}_{1}$ and $\mathrm{C}_{2}$ in Fig. 2(a)]. The depression often appears repeatedly on a weak spot, at a pressure difference possibly lower than $\Delta P_{C}[25,26]$. If $\Delta P$ is then decreased back to 0 , another stable branch is followed, along which the radius of the depression decreases progressively much below $R$ [D in Fig. 2(a)]. Then, a small amplitude unbuckling instability brings the shell shape back to the isotropic branch.

Shape hysteresis and shape dynamics.-The evolution in the height $H$ and width $W$ of the shell during a pressure cycle exhibits the hysteresis that is a necessary condition for swimming at low Reynolds number [Fig. 2(a)].

After buckling, shape oscillations of frequency $\omega$ can be observed in most liquids [Fig. 2(b)]. We define the buckling velocity $V_{b}$ as $2 \max (|d H / d t|)$, which should be close to the maximum velocity of the buckling spot. Figure 2(c) shows that this velocity is almost constant for fluid viscosities up to $\simeq 1 \mathrm{Pas}$, after which the influence of fluid damping on the shell dynamics cannot be neglected.

Displacements.-Displacements as a function of fluid viscosity are shown in Fig. 3, for both phases of the cycle (increase or decrease of $\Delta P$ ), and three different relative thicknesses $d / R$.

At low fluid Reynolds number [in Ucon oil of viscosity $37 \mathrm{~Pa} \mathrm{~s}, \operatorname{Re} \equiv \rho_{f} V_{b}(R+d / 2) / \eta=0.7$ for $\left.d / R=0.22\right]$, displacements are important in both phases (deflation and reinflation), but they almost compensate within one cycle, with a final displacement of around $1 \%$ of the radius due to shape hysteresis (Fig. 1). In this Stokes regime, the displacement is quite similar for all shell thicknesses, as is the sequence of shapes.

On the opposite end of the viscosity range $\left(\operatorname{Re}=6.10^{4}\right.$ in water), the inertial thrust should scale like $\rho_{f} \Delta \mathcal{V} \times V_{b} \omega$, where $\rho \Delta \mathcal{V}$ is the mass of the accelerated fluid in the
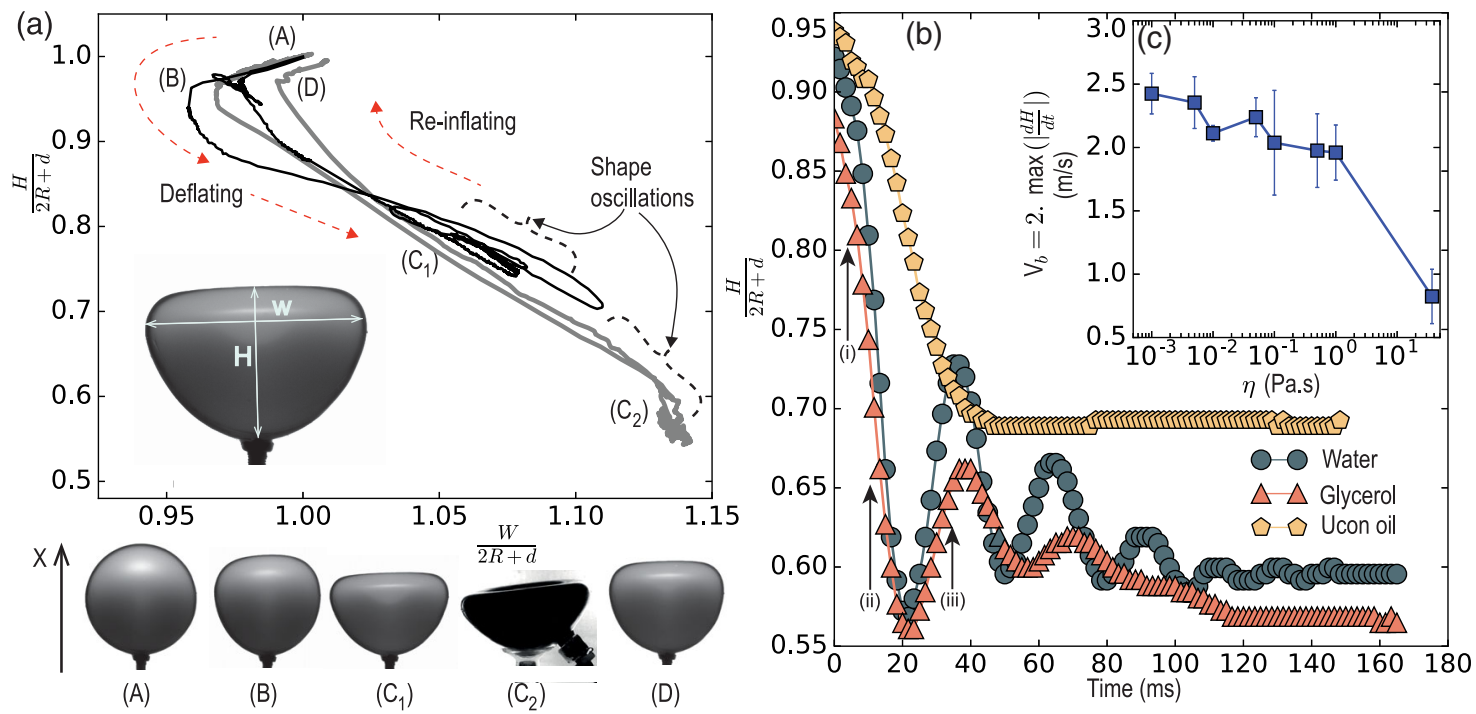

FIG. 2. Buckling and deformation of a shell with $d / R=0.22$. (a) Path in a diagram of height $H$ and width $W$ along one pressure cycle in glycerol. Pressure difference $\Delta P$ is tuned by acting either on the internal pressure (thick gray curve), or on the external pressure (thin black curve). Pressure cycles of amplitude 0-600 mbar and period $15 \mathrm{~s}$ were applied. Pictures show the different shapes met by the shell along the pressure cycle: A, initial spherical shape; $\mathrm{B}$, deformed shape right before buckling; $\mathrm{C}_{1}$, buckled shape in the case of external pressure control (encapsulated air prevents full collapse); $\mathrm{C}_{2}$, buckled shape in the case of internal pressure control; $\mathrm{D}$, shape after wrinkle unfolding right before unbuckling. (b) Time evolution of the height $H /(2 R+d)$ right after buckling [around configuration $\mathrm{C}_{2}$ in panel (a)] in three different liquids: water $\left(\eta=10^{-3}\right.$ Pa s), glycerol ( $\eta=0.9$ Pa s), Ucon@ o oil ( $\eta=37$ Pa s). (c) Buckling velocity $V_{b}$ as a function of fluid viscosity $\eta$. 

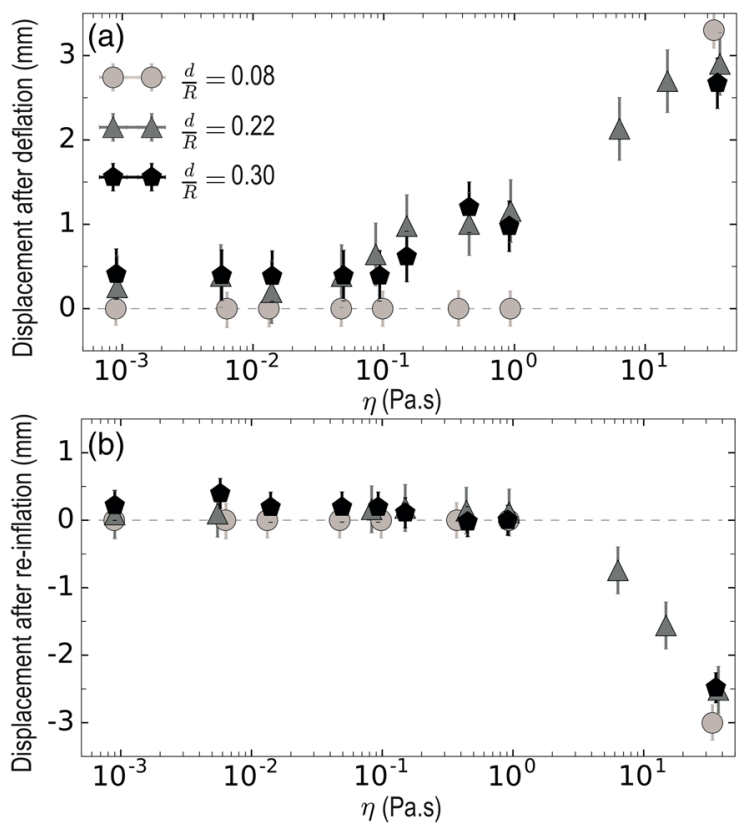

FIG. 3. Same as in Fig. 1, but decomposed into displacement during deflation and displacement during reinflation.

vicinity of the (un)buckling area and $V_{b} \omega$ its typical acceleration.

In our macroscopic model, this thrust serves first to accelerate the whole system (ball + gliding support) of mass $M \simeq 1.5 \mathrm{~kg}$. For the $d / R=0.22$ shell, where $\Delta \mathcal{V}$ is of order $20 \%$ of the total volume $\mathcal{V}_{0}$ [19], this results in a typical swimming velocity $V_{s}=V_{b} \times 0.2 \rho_{f} \mathcal{V}_{0} / M \simeq$ $0.02 \mathrm{~m} / \mathrm{s}$, in very good agreement with the velocity measured during buckling [Fig. 4(b)].

The accelerated volume $\Delta \mathcal{V}$ is an increasing function of $d / R$ [19], which makes the inertial regime eventually more efficient than the Stokes regimes for thick enough shells (Fig. 1). Interestingly, this efficiency is also a consequence of the shape hysteresis: the pre-unbuckling shape [D in
Fig. 2(a)], which is obtained after a slow decrease of the depression, is much closer to the spherical shape than the postbuckling shape [C in Fig. 2(a)]. This leads to a much smaller amount of accelerated fluid at unbuckling and, hence, a negligible contribution of the reinflation phase to the motion (Fig. 3). For instance, for the $d / R=0.22$ shell, the unbuckling volume change and velocity are smaller by a factor of 8 and 2, respectively [19].

The displacement after one full cycle appears to be even larger at an intermediate Reynolds number, for which the displacement due to buckling is enhanced. This points to the need for better knowledge of the surrounding fluid hydrodynamics.

Flows.-Flows around a $d / R=0.22$ shell attached to a fixed support were studied during buckling by timeresolved particle imaging velocimetry. They are qualitatively different according to the liquid viscosity [Fig. 4(a)], which echoes distinct evolutions of the displacement during shape oscillations [Fig. 4(b)].

In water, the flow reverses during inward and outward shell oscillations, in phase with the boundary conditions. The buckling induces a displacement of the moving support that oscillates transiently in a synchronous way with the shape.

In glycerol, the viscous effect that is not fully negligible $(\operatorname{Re}=70)$ induces a qualitatively different scenario. The collapse of the shell during phase (ii) (backward motion of both back and front ends) creates a shear flow near the translating flank. This tangential flow is still present during the outward oscillation (iii). As a result, the outward motion of the expelled fluid is not directed towards the $x$ axis. This fluid does not contribute to the inertial thrust, which lowers the backward displacement [see phase (iii) in Fig. 4(b)]. Thus, displacements due to oscillations do not counter each other as in water, but contribute, thanks to this delay effect, to a displacement lasting more than $100 \mathrm{~ms}$ [Fig. 4(b)].

The delay effect is characterized by the Womersley number Wo, defined as $\mathrm{Wo}^{2}=R^{2} \rho_{f} \omega / \eta$, which compares

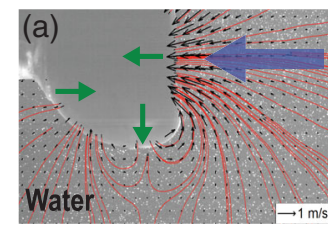

(i)

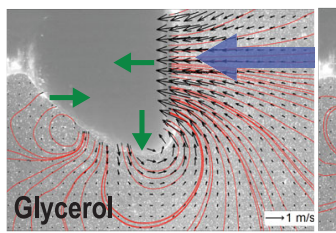

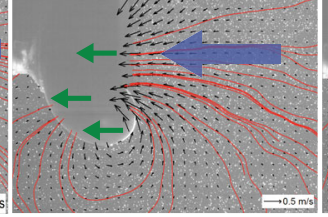

(ii)

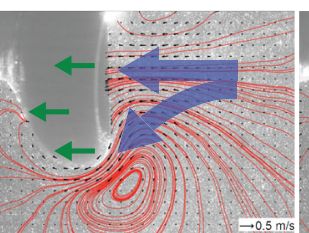

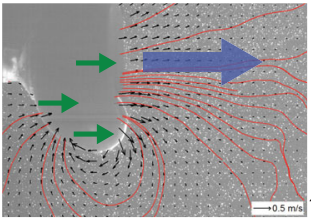

(iii)

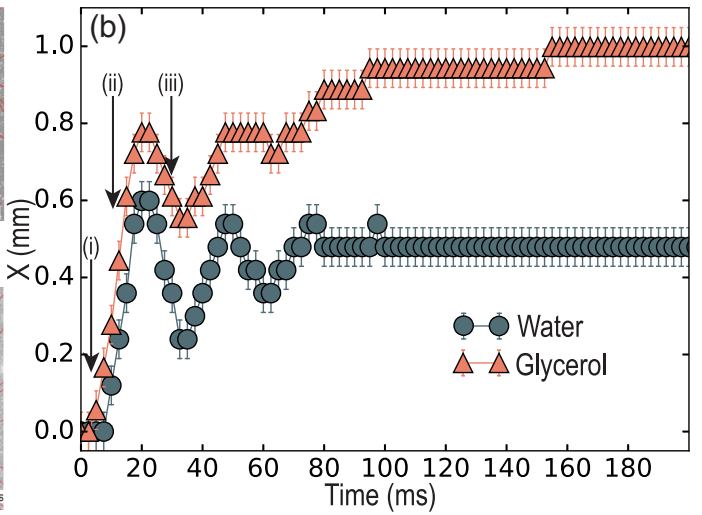

FIG. 4. Postbuckling deformations and displacements for a shell with $d / R=0.22$. (a) Stream lines and velocity field during the shape oscillations, in water and in glycerol. Blue arrows indicate the main flow patterns. Arrows inside the ball indicate the main deformation direction. Steps (i)-(iii) are indicated on the deformation curve of Fig. 2(b): (i) and (ii) correspond to the initial inwards collapse and (iii) to the first outward oscillation. (b) Displacement as a function of time. 
the oscillation period with the viscous damping time. In the classical Stokes problem of a plate oscillating with frequency $\omega$ in a viscous liquid, shear waves propagate in the normal $z$ direction with a wave number $k$ and a damping $e^{-k z}$, where $k=\sqrt{\rho_{f} \omega /(2 \eta)}$ [27]. At a given distance $R$, the shear waves are damped at high Wo and they are in phase with the plate at low Wo. For intermediate values of $\mathrm{Wo}_{\mathrm{O}} \simeq 1$, the waves are neither damped nor synchronized. In our case, the phase shift gives rise to the complex pattern observed in glycerol where $\mathrm{Wo}^{2} \mathrm{8}$, while in water $\mathrm{Wo} \simeq 300$. Since $V_{b} \simeq R \omega, \mathrm{Re} \simeq \mathrm{Wo}^{2}$ in our configuration; thus, the interplay between inertial, nonstationary dynamics and viscous damping of shear waves will always take place at an intermediate-Reynolds-number regime, and will imply an enhancement of the thrust during deflation.

Discussion and miniaturization.-We discuss the implications of our results for the motion of a colloidal armored bubble in a waterlike fluid, for which the control of the pressure difference would be attained by an external acoustic field.

Toy model.- To that purpose, we developed a simplified model to describe the postbuckling dynamics of the shell [28]. If we consider a viscoelastic Voigt material of loss and storage modulus $E^{\prime \prime}(\omega)$ and $E^{\prime}(\omega)$, with $E^{\prime \prime}(\omega)<E^{\prime}(\omega)$ (which, in practice, is almost always the case [30]), and if the surrounding fluid does not influence the shell dynamics, the postbuckling frequency obeys

$$
\omega \simeq \zeta \frac{1}{R} \frac{d}{R} \sqrt{\frac{E^{\prime}(\omega)}{\rho}},
$$

with $0.3<\zeta<0.4$. Here, $\rho$ holds for the shell material volume mass. The buckling speed $V_{b}$ obeys

$$
V_{b} \simeq \chi \frac{d}{R} \sqrt{\frac{E^{\prime}(\omega)}{\rho}},
$$

with $0.4<\chi<0.9$. Note that by dimensionality arguments $V_{b}$ necessarily scales like $f(d / R) \sqrt{E^{\prime} / \rho}$.

For our $d / R=0.22$ shell with $\rho=1060 \mathrm{~kg} / \mathrm{m}^{3}$, if we assume that $E^{\prime}(\omega)=E^{\prime}(0) \equiv E$, we find $\omega \simeq 64 \mathrm{~Hz}$; this is comparable the measured pulsation $\omega \simeq 150 \mathrm{~Hz}$ and also validates the above assumption since, for elastomeric materials, the stiffness $E^{\prime}(\omega)$ is almost constant up to the $\mathrm{kHz}$ range [30]. We find that the buckling speed $V_{b}$ can be composed of values between 1.9 and $4.4 \mathrm{~m} / \mathrm{s}$, which perfectly surrounds the experimental value of $V_{b} \simeq 2.4 \mathrm{~m} / \mathrm{s}$ in water.

This validates Eqs. (1) and (2), which allows us to estimate the buckling velocities and postbuckling frequency from the sole knowledge of $E^{\prime}$ without that of $E^{\prime \prime}$. This opens discussion for possible scalings between microscopic and macroscopic systems.

Flow regime.-From the preceding calculation, we expect the Reynolds number to be $\operatorname{Re} \simeq 0.4(R / \eta)\left(\rho \Delta P_{C}\right)^{1 / 2}$, with a prefactor $\simeq \sqrt{E^{\prime}(\omega) / E}$. Displacement enhancement is controlled by the Womersley number; in this problem, $\mathrm{Wo}_{\mathrm{O}} \simeq \mathrm{Re}^{1 / 2}$.

For a shell of radius $10 \mu \mathrm{m}$, and considering for $\Delta P_{c}$ the maximum value of 1 bar to avoid cavitation by the \pm 1 bar pressure wave, $\operatorname{Re} \simeq 40 \sqrt{E^{\prime}(\omega) / E}$. Equation (1) shows that, as $\omega$ scales as $R^{-1}$, miniaturization down to $10 \mu \mathrm{m}$ propels $\omega$ to the MHz range. Usual values of compliance for elastomers [30] indicate that Re may reach 400. Microscopic shells could then swim in the 1-600 intermediate Re regime, where inertial thrust is enhanced by the coupling between flow and shape oscillations. This happens at frequencies compatible with sonographic devices that are already known to induce repeated buckling on armored bubbles [21].

Expected displacements.-A microscopic shell would be controlled by variations of the external pressure, while our macroscopic model was activated by varying the internal pressure.

At low Reynolds number, the sequence of shapes is quasisimilar for both ways of controlling the pressure difference [Fig. 2(a)] so we anticipate our result of a displacement per cycle of $1 \%$ of $R$ (which is a slight underestimation due to friction on the arm holding the swimmer) to also hold for a microscopic shell.

At higher Reynolds number, the mass of accelerated fluid is given by the loss of shell volume during buckling, which is limited by the resistance of the inner gas to compression. However, it can be shown that to the first two orders in $d / R$, the lost volumes are identical whatever the way the pressure is controlled [19]. The estimate done for the final displacement in the intermediate $\operatorname{Re}$ (and Wo) regime $1<\operatorname{Re}<600$ is then valid, and is even a minor bound for a microswimmer that would not be attached to a heavy support.

Finally, a microswimmer subjected to an ultrasonic wave of amplitude $\Delta P_{C}$ and driving frequency $\omega_{d}$ will swim under the condition that $\omega_{d}<\omega$, where $\omega$ is the postbuckling spontaneous frequency of the shape oscillations, so as to allow time for the material to react to pressure variations. While the displacement per cycle is rather low, the high frequency that is allowed, thanks to the fast activation due to the instability, may lead to high velocities. We find that swimming velocity is at least equal to $U_{s}=0.01 R \times$ $\omega /(2 \pi)$ (the Stokes case). With $\omega_{d} \simeq \omega \simeq 1 \mathrm{MHz}$, this potentially leads to a net velocity $\sim 1500 R$ per second, which is $15 \mathrm{~mm} / \mathrm{s}$ for a $R=10 \mu \mathrm{m}$ shell, much faster than that of Janus particles $(10 \mu \mathrm{m} / \mathrm{s}$ [31]), helicoidal microrobots $(10 \mu \mathrm{m} / \mathrm{s}$ [10]), microrobots with acoustically activated flagella $(50 \mu \mathrm{m} / \mathrm{s}$ [12] to $1 \mathrm{~mm} / \mathrm{s}$ but for much larger swimmers [15]), and microrobots propelled by metachronal waves $(3 \mu \mathrm{m} / \mathrm{s}$ [32]).

A microscopic shell designed in such a way that the buckling pressure is of the order 1 bar would be in the intermediate regime and would even swim faster. In addition, we anticipate that the swimming amplification observed in the moderate-Womersley-number regime opens a path for active 
amplification by a fine-tuning of the pressure cycle period, so as to make it comparable to the viscous decay time.

Finally, we extrapolate that multidirectional remote control in a compound of several spheres of different characteristics, which may be built using smart self-assembly properties of colloidal particles [33-35], can be reached by playing on the wave amplitude (with strong nonlinear onoff response depending on whether the buckling pressure has been reached or not) and/or on the wave frequency.

Conclusion.-We have proposed and experimentally tested a swimming mechanism active at all Re numbers, which relies on the intrinsic property of shape deformation hysteresis of a spherical shell upon a deflation or reinflation cycle. Thanks to the fast deformations associated with shape instabilities, an inertial regime is reachable even at small scales. Hysteresis in the deformation velocity sequence implies fast propulsion in this regime, which can be amplified by the coupling between shape oscillations and flow patterns.

This research has received funding from the European Research Council under the European Union's Seventh Framework Programme (FP7/2007-2013)/ERC Grant No. 614655 Bubbleboost. We thank C. Grégoire for his contribution as an internship student, L. Vignale and N. Mordant for their help and advice for the PIV, T. Combriat, P. Peyla, S. Rafaï, and T. Podgorski for the numerous discussions, O. Stephan and S. Lecuyer for their help in the shellmolding process, and all the workshop employees for their commitment to build up the different experimental setups.

* Corresponding author. gwennou.coupier@univ-grenoble-alpes.fr

[1] H. A. Stone and A. D. T. Samuel, Phys. Rev. Lett. 77, 4102 (1996).

[2] F. Alouges, A. DeSimone, and A. Lefebvre, J. Nonlinear Sci. 18, 277 (2008).

[3] J. E. Avron, O. Gat, and O. Kenneth, Phys. Rev. Lett. 93, 186001 (2004).

[4] A. Farutin, S. Rafaï, D. K. Dysthe, A. Duperray, P. Peyla, and C. Misbah, Phys. Rev. Lett. 111, 228102 (2013).

[5] K. Ishimoto and E. A. Gaffney, Phys. Rev. E 90, 012704 (2014).

[6] D. Walker, M. Kübler, K. I. Morozov, P. Fischer, and A. M. Leshansky, Nano Lett. 15, 4412 (2015).

[7] N. G. Chisholm, D. Legendre, E. Lauga, and A. S. Khair, J. Fluid Mech. 796, 233 (2016).

[8] A. Najafi and R. Golestanian, Phys. Rev. E 69, 062901 (2004).

[9] R. Dreyfus, J. Baudry, M. L. Roper, M. Fermigier, H. A. Stone, and J. Bibette, Nature (London) 437, 862 (2005).
[10] K. Peyer, A. Mahoney, L. Zhang, J. Abbott, and B. Nelson, Bacteria-Inspired Microrobots (Elsevier, New York, 2012), pp. 165-199.

[11] K. E. Peyer, S. Tottori, F. Qiu, L. Zhang, and B. J. Nelson, Chem. Eur. J. 19, 28 (2013).

[12] D. Ahmed, T. Baasch, B. Jang, S. Pane, J. Dual, and B. J. Nelson, Nano Lett. 16, 4968 (2016).

[13] T. Qiu, S. Palagi, and P. Fischer, in 2015 37th Annual International Conference of the IEEE Engineering in Medicine and Biology Society (EMBC) (IEEE, New York, 2015), pp. 4922-4925.

[14] N. Bertin, T. A. Spelman, O. Stephan, L. Gredy, M. Bouriau, E. Lauga, and P. Marmottant, Phys. Rev. Applied 4, 064012 (2015).

[15] M. Kaynak, A. Ozcelik, A. Nourhani, P. E. Lammert, V. H. Crespi, and T. J. Huang, Lab Chip 17, 395 (2017).

[16] E. M. Purcell, Am. J. Phys. 45, 3 (1977).

[17] C. Zoldesi and A. Imhof, Adv. Mater. 17, 924 (2005).

[18] E. Pisani, C. Ringard, V. Nicolas, E. Raphaël, V. Rosilio, L. Moine, E. Fattal, and N. Tsapis, Soft Matter 5, 3054 (2009).

[19] See Supplemental Material at http://link.aps.org/ supplemental/10.1103/PhysRevLett.119.224501 for the detail of the experimental setup, for the calculation of the final volumes after buckling and for additional data on the unbuckling dynamics.

[20] S. Knoche and J. Kierfeld, Phys. Rev. E 84, 046608 (2011).

[21] P. Marmottant, A. Bouakaz, N. de Jong, and C. Quilliet, J. Acoust. Soc. Am. 129, 1231 (2011).

[22] J. W. Hutchinson, J. Appl. Mech. 34, 49 (1967).

[23] L. Landau and E. Lifschitz, Theory of Elasticity, 3rd ed. (Elsevier Butterworth-Heinemann, Oxford, 1986).

[24] C. Quilliet, Eur. Phys. J. E 35, 48 (2012).

[25] A. Lee, F. López Jiménez, J. Marthelot, J. Hutchinson, and P. M. Reis, J. Appl. Mech. 83, 111005 (2016).

[26] L. Zhang and C. Q. Ru, Phys. Rev. E 93, 062403 (2016).

[27] G. Batchelor, An Introduction to Fluid Dynamics (Cambridge University Press, Cambridge, England, 1967).

[28] See Supplemental Material at http://link.aps.org/ supplemental/10.1103/PhysRevLett.119.224501 for a description of the model, based on the theory of viscoelastic thin rods [29].

[29] W. E. Baker, W. E. Woolam, and D. Young, Int. J. Mech. Sci. 9, 743 (1967).

[30] R. S. Lakes, Viscoelastic Materials (Cambridge University Press, Cambridge, England, 2009).

[31] W. F. Paxton, K. C. Kistler, C. C. Olmeda, A. Sen, S. K. St. Angelo, Y. Cao, T. E. Mallouk, P. E. Lammert, and V. H. Crespi, J. Am. Chem. Soc. 126, 13424 (2004).

[32] S. Palagi, E. W. Jager, B. Mazzolai, and L. Beccai, Bioinspiration Biomimetics 8, 046004 (2013).

[33] S.-M. Yang, S.-H. Kim, J.-M. Lim, and G.-R. Yi, J. Mater. Chem. 18, 2177 (2008).

[34] L. Cademartiri, K. J. M. Bishop, P. W. Snyder, and G. A. Ozin, Phil. Trans. R. Soc. A 370, 2824 (2012).

[35] G.-R. Yi, D. J. Pine, and S. Sacanna, J. Phys. Condens. Matter 25, 193101 (2013). 